\title{
A comparison of the NEIVFQ25 and GQL-15 questionnaires in Nigerian glaucoma patients
}

This article was published in the following Dove Press journal:

Clinical Ophthalmology

3I August 2012

Number of times this article has been viewed

\section{Chigozie A Mbadugha \\ Adeola O Onakoya \\ Olufisayo T Aribaba \\ Folashade B Akinsola}

Guinness Eye Centre, Department of Ophthalmology, Lagos University Teaching Hospital, Lagos State, Nigeria

Video abstract

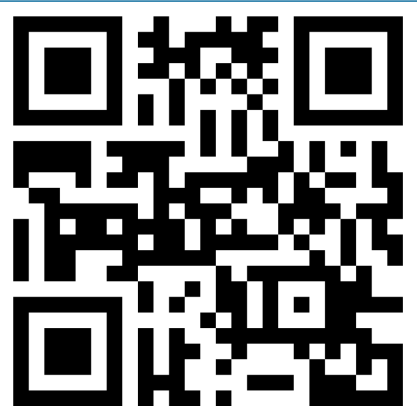

Point your SmartPhone at the code above. If you have a QR code reader the video abstract will appear. Or use: http://dvpr.es/NdOIG6
Correspondence: CA Mbadugha Guinness Eye Centre, Department of Ophthalmology, Lagos University Teaching Hospital, Lagos, Nigeria

Tel +2347069596172

Fax +234I46237I 5

Email cmbadugha@yahoo.co.uk
Aim: To compare two vision-specific quality of life (QOL) instruments - the disease-specific 15-item Glaucoma Quality of Life questionnaire (GQL-15) and the nonglaucoma-specific 25-item National Eye Institute Visual Function Questionnaire (NEIVFQ25).

Methods: The QOL of 132 glaucoma patients being managed in Lagos University Teaching Hospital and an equal number of controls matched for age and sex was assessed using two vision-specific instruments: GQL-15 and the NEIVFQ25. The categorization of the severity of glaucoma into mild, moderate, and severe disease was determined using the degree of visual field loss. The Statistical Package for the Social Sciences (version 15; SPSS Inc, Chicago, IL) software program was used for analyzing the data obtained. Spearman's correlation coefficients were used to assess the correlation between the scores from the two questionnaires.

Results: Patients had the greatest difficulty with activities affected by glare and dark adaptation in the GQL-15. Driving and general vision were the factors most affected in the NEIVFQ25. The Spearman rho values showed strong correlations (rho $>0.55$ ) between the NEIVFQ25 and GQL-15 QOL scores for the total number of participants (rho: -0.75 ), total number of cases (rho: -0.83 ), and the mild (rho: -0.76 ), moderate (rho: -0.75 ), and severe (rho: -0.84 ) cases. There was a moderate correlation (rho: -0.38 ) for QOL scores of controls. Cronbach's alpha was 0.94 for the GQL-15 and 0.93 for the NEIVFQ25, showing high internal consistency for both questionnaires.

Conclusion: The GQL-15 and the NEIVFQ25 questionnaires showed high internal consistency, correlated strongly with each other, and were reliable in the assessment of glaucoma patients in this study.

Keywords: primary open-angle glaucoma, quality of life questionnaires, GQL-15, NEIVFQ25

\section{Introduction}

The term "quality of life" (QOL) is the subjective perception of well-being and wholeness. It is a broad concept affected in a complex way by a person's physical health, psychological state, level of independence, social relationships, and his or her relationship to the salient features of his or her environment. ${ }^{1}$ It reflects the difference between a person's hopes and expectations and his or her present experience. Thus, QOL is influenced by the social, health, economic, and environmental conditions that affect human and social development.

The main goal of treatment in glaucoma patients is to improve clinical outcomes thereby improving the patient's QOL. ${ }^{2}$ Measures of visual acuity (VA), intraocular pressures (IOP), and visual fields have been utilized over the years to assess treatment efficacy in glaucoma. ${ }^{3}$ The achievement of therapeutic goals based on clinical indicators 
and parameters is important, but naturally, patients are not interested in improvements in clinical indicators unless they translate to an improvement in their QOL. ${ }^{4}$ In addition, these clinical tests do not measure an individual's ability to function in the everyday environment. ${ }^{5}$ There is a growing demand to include subjective patient-based visual function assessments in the measurement of treatment outcomes, as patient perception is being increasingly recognized as the central determinant in monitoring the outcome of medical interventions ${ }^{6}$ and visual function assessments from the patient's perspective are being used to measure treatment outcomes.

Several generic QOL instruments have been developed and sometimes they are used with vision-related QOL instruments to assess the QOL of ophthalmic patients. This has been termed a modular approach and has been advocated for the study of vision-related $\mathrm{QOL}^{7}$; examples include the Medical Outcomes Study Short Form-36 (SF-36) ${ }^{8}$ and the Sickness Impact Profile (SIP). ${ }^{9}$ The use of generic instruments makes comparison between the effects of ophthalmic and medical conditions on QOL easier.

Vision-related QOL instruments may be disease-specific or nonspecific. The nondisease-specific vision-related QOL instruments include: the National Eye Institute Visual Function Questionnaires (NEIVFQ51, ${ }^{10}$ NEIVFQ25 ${ }^{11}$ ), the 14-item Visual Functioning Index (VF-14), ${ }^{12}$ Assessment of Function Related to Vision (AFREV), ${ }^{13}$ the Activities of Daily Vision Scale (ADVS), ${ }^{14}$ and the Visual Activities Questionnaire (VAQ). ${ }^{15}$ Quality of life assessment using a nondisease-specific instrument facilitates the direct comparison of the QOL of patients with different eye problems.

Some specific QOL instruments have been developed for glaucoma, such as the Glaucoma Quality of Life 15 (GQL-15), ${ }^{16}$ the Symptom Impact Glaucoma Score (SIG), ${ }^{17}$ the Glaucoma Health Perceptions Index (GHPI), ${ }^{17}$ the Glaucoma Symptom Scale (GSS). ${ }^{18}$ and the Comparison of Ophthalmic Medication for Tolerability (COMTOL). ${ }^{19}$ These instruments are thought to be more accurate and specific for glaucoma with more visual field considerations. ${ }^{20}$

The NEIVFQ25 is a questionnaire developed by the Research and Development Corporation. It is a shorter version of the longer questionnaire, which has 51 items (NEIVFQ51), with reduced administration time. Studies suggest that the NEIVFQ is a reliable tool for the evaluation of QOL in glaucoma patients. ${ }^{21,22}$ As it is a broader instrument used to assess all visual problems, newer and disease-specific tools can be compared to it.
The NEIVFQ25 has five nonvisual domains: general health, mental health, dependency, social function, role limitations; and seven visual domains: general vision, distance vision, peripheral vision, driving, near vision, color vision, and ocular pains.

The GQL-15 is a 15-item questionnaire designed to take into account the effect of binocular visual field loss on visual function. ${ }^{16}$ It is made up of four domains: outdoor mobility, peripheral vision, near vision, and glare and dark adaptation. This questionnaire is shorter than the NEIVFQ25, and therefore, easier to use and faster to administer. It has been shown to be reliable and has good internal consistency. ${ }^{23}$ The instrument, however, focuses mainly on vision-related difficulty and does not address other issues, such as mental health, role difficulty, dependency, or general health, as does the NEIVFQ25.

The NEIVFQ25 (a nonglaucoma-specific questionnaire) and the GQL-15 (a glaucoma-specific questionnaire) are compared in this study with a view to assessing their correlation in this group of participants and to determine which of the instruments is better suited to assess QOL issues in Nigerian glaucoma patients, given the paucity of data on QOL for this population. The focus of this study was on primary open-angle glaucoma (POAG), which is the most common variant of glaucoma in Nigeria. ${ }^{24-26}$

\section{Methods}

Two hundred sixty-four participants were recruited (132 cases and 132 controls) for the present study. Consecutive patients with POAG and corrected refractive errors attending the Guinness Eye Centre, Lagos University Teaching Hospital were recruited as cases and controls, respectively. The enrolment strategy aimed to match the cases and controls by 5 -year age categories and by sex. One hundred thirty-two (132) patients with a confirmed diagnosis of POAG were enrolled in the study. There were three groups of 44 participants each, based on severity of disease (mild, moderate, and severe). Ethical approval was obtained from the institutional ethical committee. All participants provided written informed consent and the declaration of Helsinki (October 2008 revision) was adhered to. Participants were free to withdraw from the study at any time and their decision did not affect any aspect of the care they were receiving from the institution.

POAG was defined as the presence of glaucomatous optic nerve head changes, open anterior chamber angles, and visual field loss with or without an elevated IOP in one or both eyes. A thorough comprehensive ocular examination 
was carried out on all participants; this examination included gonioscopy, dilated stereoscopic optic nerve head assessment at the slit-lamp with a Volks +78 diopter sphere (DS) lens, and standard achromatic perimetry using a full threshold central 30-2 strategy (Optifield Sinemed lnc, Benicia, CA).

Visual field defects were classified into mild, moderate, and severe visual field loss using mean deviation (MD) indices and a modification of the Hodapp-Parrish-Anderson classification ${ }^{27}$ in the more severely affected eye. Mild visual field loss was defined as a mean deviation of less than or equal to -6 decibels $(\mathrm{dB})$, moderate visual field loss was defined as $\mathrm{MD}$ greater than $-6 \mathrm{~dB}$ but less than $-12 \mathrm{~dB}$, and severe visual field defect as MD greater than $-12 \mathrm{~dB}$.

\section{Eligibility criteria for cases}

Patients with POAG on medical therapy or those who had had trabeculectomy at least 3 months before the study were eligible. The purpose of the 3-month period was to ensure that the surgical experience was not recent and would not significantly influence QOL responses.

\section{Exclusion criteria for cases}

The presence of refractive errors greater than 5 DS or 2 diopters Cylinder (DCyl), visually significant cataracts (greater than Stage 2 LOCS III classification), ${ }^{28}$ or any other ocular pathology were criteria for exclusion from the study. Patients with secondary glaucoma or angleclosure glaucoma were also excluded. All patients who had undergone trabeculectomy, or any intraocular surgery, within the 3 months preceding the study were excluded from the study. Patients with mental or systemic illness, and hearing, cognitive or mobility defects were excluded from the study.

\section{Eligibility criteria for controls}

Age and sex-matched patients with refractive errors corrected to at least 6/9, normal optic discs and visual fields, and no underlying ocular pathology or systemic co-morbidities were recruited as controls. The degree of refractive error acceptable was less than 5 DS from emmetropia or less than 2 DCyl of astigmatism.

\section{Exclusion criteria for controls}

Patients with suspicious optic discs, abnormal visual fields, ocular hypertension (defined as $1 \mathrm{OP}$ greater than $21 \mathrm{mmHg}$ in either eye), visually significant lens opacities, high refractive errors, and corrected visual acuity less than $6 / 9$ were excluded. Participants who had a history of glaucoma or unexplained blindness in a first-degree relative or who were being managed for systemic illness were also excluded from the study.

\section{Quality of life assessment}

Quality of life assessment was done using the intervieweradministered format of the NEIVFQ25 and the GQL-15 questionnaires. Two optional utility questions rating general health and vision on a linear scale from the NEIVFQ25 appendix were included in the questionnaire. The NEIVFQ25 was administered first, followed by the GQL-15, administered by the same interviewer in a single sitting. Participants were not informed that two different QOL instruments were being administered.

\section{Translation of QOL assessment questionnaires}

For respondents who were not literate in English, the NEIVFQ25 and the GQL-15 questionnaires were translated into Yoruba, Igbo, and Hausa, which are the three most prominent indigenous Nigerian languages. Care was taken to reduce any bias that could occur because of translation. Translation and back-translation methods were used by competent bilingual translators to ensure accuracy.

The scoring method designed by the Research and Development Corporation for the NEIVFQ25 was used. Individual items were scored. The minimum score was zero and the maximum score for each item was 100. A higher score signified a better QOL. The average score for each domain was calculated from the individual item scores. The summary NEIVFQ25 score was obtained by calculating the average of the domain scores with the exclusion of the general health domain score.

With the GQL-15, higher scores indicate poorer QOL. A scale of 0 to 5 was used to code the item level responses: 5 represented severe difficulty due to visual reasons, 1 indicated no difficulty with performing the activity, and 0 signified abstinence from activity for nonvisual reasons. Item response scores of the GQL-15 were added to obtain the summary scores.

To calculate the subscale scores for the four domains of the GQL-15, the item level responses were scored on a numerical interval scale ranging from 0 , indicating no difficulty, to 100 , indicating severe difficulty. The subscale score for each domain was calculated using an average of the scores generated for the component itemlevel responses. Poorer QOL and increasing difficulty with vision-related activities were associated with higher subscale scores. 
A measure of the degree of difficulty in performing visual tasks outlined in the GQL-15 was depicted by corresponding visual performance. ${ }^{16}$ This score was obtained by first subtracting the subscale scores from the total possible score $\times 100(1500)$. The product was thereafter converted to a percentage.

\section{Data entry and statistical analysis}

Data was entered into Microsoft Excel for Windows XP Professional and was later uploaded and analyzed using the Statistical Package for the Social Sciences version 15 (SPSS, Chicago III). Cronbach's alpha was used to assess the internal consistency of the questionnaires. The range, mean, median, and standard deviation of QOL scores for the two questionnaires were calculated and compared for the subgroups of participants. Spearman's rank correlation coefficients were used to assess the correlation between the GQL-15 and the NEIVFQ25. A modest or weak correlation was defined as rho values $<0.31$, values ranging from $0.32-0.55$ were defined as moderate correlation, and rho values $>0.55$ showed strong correlation. $P$-values equal to or less than 0.05 were considered statistically significant.

\section{Results}

Administration of the NEIVFQ25 questionnaire took about 12 minutes, on average, while administration of the GQL-15 lasted about 5 minutes. The Cronbach's alpha for the NEIVFQ25 was 0.93, and 0.94 for the GQL-15. Eight participants (3\%) responded to the translated versions of the NEIVFQ25 and GQL-15 questionnaires. Mild glaucoma patients had a mean age of 52.81 years. For patients with moderate and severe glaucoma, the mean age was 59.11 and 62.67 years, respectively. Most of these patients had bilateral disease (90.9\%), and binocular visual field defects were demonstrated in $93.94 \%$ of the patients.

\section{NEIVFQ25 and GQL-15 scores}

\section{in cases and controls}

The mean QOL score using the NEIVFQ25 was higher in controls, 96.7 ( $\mathrm{SD} \pm 2.34$ ), than in cases, 85.2 ( $\mathrm{SD} \pm 16.07)$. Using the GQL-15, the mean QOL for controls was 15.75 $(\mathrm{SD} \pm 1.85)$ and $24.07(\mathrm{SD} \pm 12.4)$ for cases. The corresponding visual performance was $84.54 \%$ ( $\mathrm{SD} \pm 21.65$ ) for cases and $98.75 \%$ ( $\mathrm{SD} \pm 3.02$ ) for controls (see Figure 1). All these differences had a $P$-value of $<0.001$ and thus were statistically significant. Females, younger participants, and participants who had received tertiary education reported better QOL with both the GQL-15 and the NEIVFQ25.

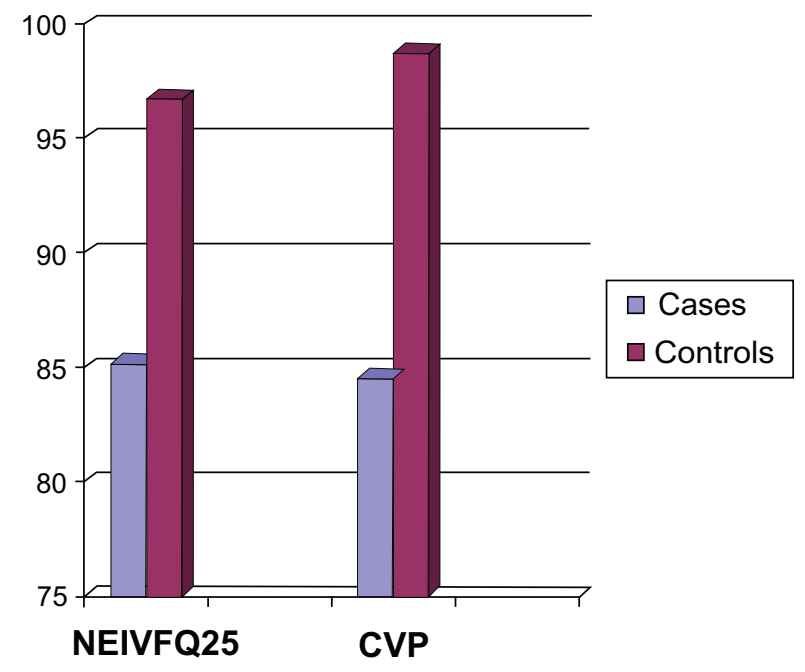

Figure I Mean NEIVFQ25 QOL score and CVP of cases and controls. Abbreviations: NEIVFQ25, 25-item National Eye Institute Visual Functioning Questionnaire; CVP, corresponding visual performance; QOL, quality of life.

\section{NEIVFQ25 and GQL-I5 scores in varying degrees of disease severity}

The average QOL scores in patients with moderate and severe glaucoma were significantly different $(P<0.001)$. This was also the case for participants with severe and mild glaucoma. This difference was noted for the two QOL questionnaires. Both questionnaires, however, did not detect a significant difference in the QOL scores of patients with mild and moderate glaucoma. A comparison of the GQL-15 QOL scores of patients with mild and moderate glaucoma using the Mann-Whitney $U$ test did not show a statistically significant difference $(P=0.25)$. A comparison of the NEIVFQ25 QOL scores for mild and moderate glaucoma was also not statistically significant $(P=0.92)$. The decrease in QOL from controls $(\mathrm{n}=132)$ across mild $(\mathrm{n}=44)$, moderate $(\mathrm{n}=44)$, and severe $(\mathrm{n}=44)$ glaucoma cases for the NEIVFQ25 and the GQL-15 are depicted in the form of line plots in Figures 2 and 3.

\section{NEIVFQ25, GQL-I5 scores}

\section{and correlation with visual function}

The NEIVFQ25 scores correlated best with the mean logarithm of the minimum angle of resolution (logMAR) Visual Acuity (VA) better eye (rho: $0.54, P<0.001$ ), mean contrast sensitivity score (rho: $0.47, P<0.0010$ and mean $\log$ MAR VA worse eye (rho: $0.46, P<0.001$ ). The GQL-15 scores correlated best with mean deviation in both eyes (MD OU), (rho: $0.43, P<0.001$ ), mean contrast sensitivity score (rho: $0.43, P<0.001$ ), and mean log MAR VA worse eye (rho: $-0.42, P<0.001$ ). Only a modest or weak correlation existed between the pattern standard deviation of 


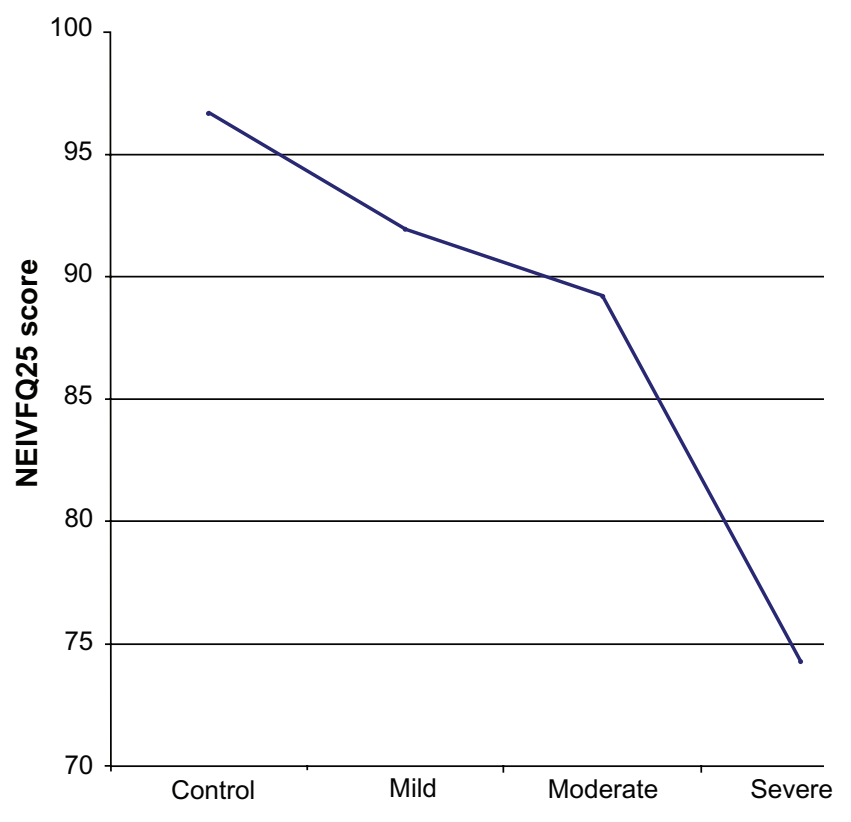

Figure 2 Line plot of mean NEIVFQ25 scores in controls, mild, moderate and severe glaucoma cases.

Abbreviation: NEIVFQ25, 25-item National Eye Institute Visual Function Questionnaire.

patients and both the NEIVFQ25 (rho: $-0.13, P=0.16$ ) and GQL-15 (rho: $0.13, P=0.15$ ).

\section{Correlation between NEIVFQ25 and GQL-I 5 scores}

Correlation between the NEIVFQ25 and GQL-15 QOL scores was assessed using Spearman's correlation coefficient. The scores had strong correlations when the scores for total

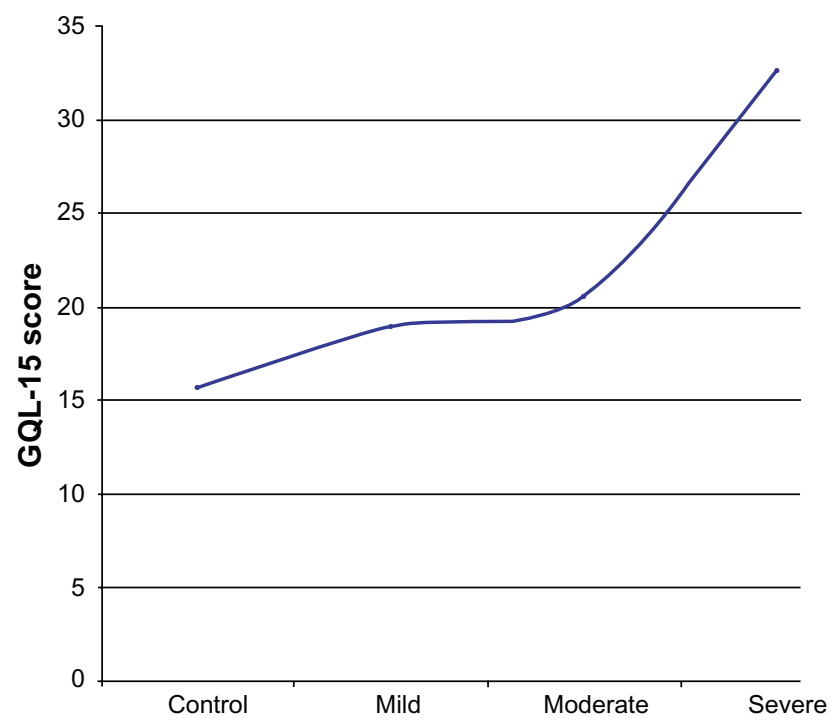

Figure 3 Line plot of mean GQL-15 scores in controls, mild, moderate and severe glaucoma cases.

Note: *Lower figures denote better QOL.

Abbreviations: GQL-15, I5-item Glaucoma Quality of Life questionnaire; QOL, quality of life. number of participants $(-0.753)$, total number of cases $(-0.827)$, and mild $(-0.762)$, moderate $(-0.748)$, and severe cases $(-0.836)$ were compared. There was a moderate correlation for QOL scores of controls with the Spearman's correlation coefficient $($ rho $=-0.378)$.

\section{Correlation between NEIVFQ25 and GQL-I5 subscales/domains}

Using the GQL-15, the glare and dark adaptation subscale was the most affected, while the NEIVFQ25 revealed that the general vision and driving subscales were the most affected. Figure 4 clearly shows that participants had the greatest difficulty with tasks affected by glare and dark adaptation.

There were statistically significant correlations between the subscales of the NEIVFQ25 and the GQL-15; these results are shown in Table 1 . The outdoor mobility domain of the GQL-15 had the highest correlation with the peripheral vision subscale of the NEIVFQ25 (rho: $-0.620, P<0.001$ ). The glare and dark adaptation domain of the GQL-15 correlated best with the distance activities subscale of the NEIVFQ25 (rho: $-0.656, P<0.001$ ). The central and near vision domain of the GQL-15 correlated best with the near activities subscale of the NEIVFQ25 (rho: $-0.731, P<0.001$ ). The peripheral vision domain of the GQL-15 correlated best with the distance activities subscale of the NEIVFQ25 (rho: $-0.709, P<0.001$ ) but also had a strong correlation

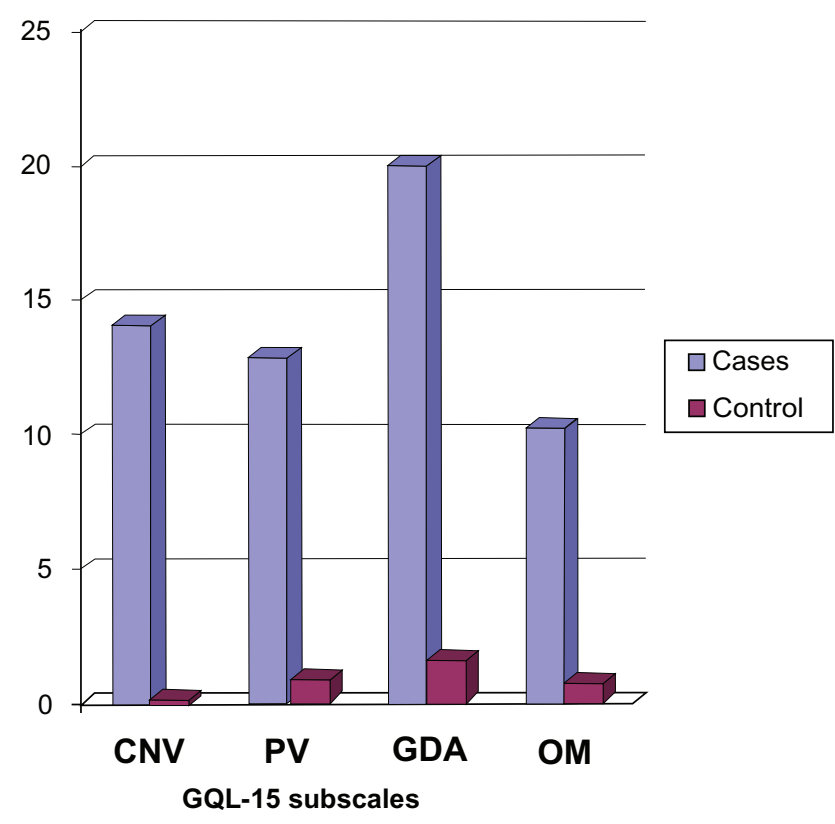

Figure 4 Bar chart showing GQL-I5 subscale scores for cases and controls*. Notes: Higher scores (bars) indicate increased difficulty with tasks.

Abbreviations: CNV, central and near vision; PV, peripheral vision; GDA, glare and dark adaptation; OM, outdoor mobility; GQL-15, I5-item Glaucoma Quality of Life questionnaire. 
Table I Correlation between NEIVFQ25 items and GQL-I5 domain items

\begin{tabular}{|c|c|c|c|c|c|}
\hline & $\begin{array}{l}\text { Outdoor } \\
\text { mobility }\end{array}$ & $\begin{array}{l}\text { Glare dark } \\
\text { adaptation }\end{array}$ & $\begin{array}{l}\text { Central and } \\
\text { near vision }\end{array}$ & $\begin{array}{l}\text { Peripheral } \\
\text { vision }\end{array}$ & $\begin{array}{l}\text { Total } \\
\text { GQL-I } 5 \text { score }\end{array}$ \\
\hline \multicolumn{6}{|l|}{ NEIVFQ25 items } \\
\hline Gen health & $-0.198 * *$ & $-0.235^{* *}$ & $-0.185^{* *}$ & $-0.280 * *$ & $-0.245^{* *}$ \\
\hline Gen vision & $-0.395^{* *}$ & $-0.447^{* *}$ & $-0.433^{* *}$ & $-0.545^{* *}$ & $-0.492 * *$ \\
\hline Ocular pain & $-0.199 * *$ & $-0.325 * *$ & $-0.305^{* *}$ & $-0.264 * *$ & $-0.348 * *$ \\
\hline Near activities & $-0.489 * *$ & $-0.59 \mid * *$ & $-0.73 I^{* *}$ & $-0.62 I^{* *}$ & $-0.635^{* *}$ \\
\hline Distance activities & $-0.510 * *$ & $-0.656 * *$ & $-0.655^{* *}$ & $-0.709 * *$ & $-0.685^{* *}$ \\
\hline Social functioning & $-0.614 * *$ & $-0.54 I * *$ & $-0.688^{* *}$ & $-0.584 * *$ & $-0.548 * *$ \\
\hline Mental Health & $-0.434 * *$ & $-0.565 * *$ & $-0.535^{* *}$ & $-0.542 * *$ & $-0.603 * *$ \\
\hline Role difficulty & $-0.614^{* *}$ & $-0.580 * *$ & $-0.618^{* *}$ & $-0.558 * *$ & $-0.577 * *$ \\
\hline Dependency & $-0.596 * *$ & $-0.508 * *$ & $-0.570 * *$ & $-0.565^{* *}$ & $-0.531 * *$ \\
\hline Driving & $-0.452 * *$ & $-0.644 * *$ & $-0.526 * *$ & $-0.488 * *$ & $-0.633 * *$ \\
\hline Colour vision & $-0.585^{* *}$ & $-0.416 * *$ & $-0.515^{* *}$ & $-0.46 I^{* *}$ & $-0.418 * *$ \\
\hline Peripheral vision & $-0.620 * *$ & $-0.520 * *$ & $-0.588^{* *}$ & $-0.639 * *$ & $-0.565^{* *}$ \\
\hline Total NEIVFQ25 score & $-0.45 \mathrm{I} * *$ & $-0.707 *$ & $-0.615^{* *}$ & $-0.668 * *$ & $-0.753 * *$ \\
\hline
\end{tabular}

Notes: Spearman's rho values. ${ }^{* *}$ Correlation significant at $0.0 \mathrm{I}$ level ( 2 tailed).

Abbreviations: NEIVFQ25, 25-item National Eye Institute Visual Functioning Questionnaire; GQL-15, I5-item Glaucoma Quality of Life questionnaire.

(rho: $-0.639, P<0.001$ ) with the peripheral vision subscale of the NEIVFQ25. The overall scores of both questionnaires correlated best with each other (rho: $-0.753, P<0.001$ ). Figure 5 is a scatter plot, graphically showing the correlation between the NEIVFQ25 and the GQL-15 scores.

\section{Discussion}

The two questionnaires had good reliability indices. The high Cronbach's alpha obtained for the NEIVFQ25 in this study (0.93) suggests internal consistency. This differs from the findings of Mangione et $\mathrm{al}^{11}$ in which a Cronbach's alpha ranging from 0.71 to -0.85 was obtained. We obtained a Cronbach's alpha of 0.94 for the GQL-15 which is only slightly higher than

Scatterplot of NEIVFQ25 and GQL-15

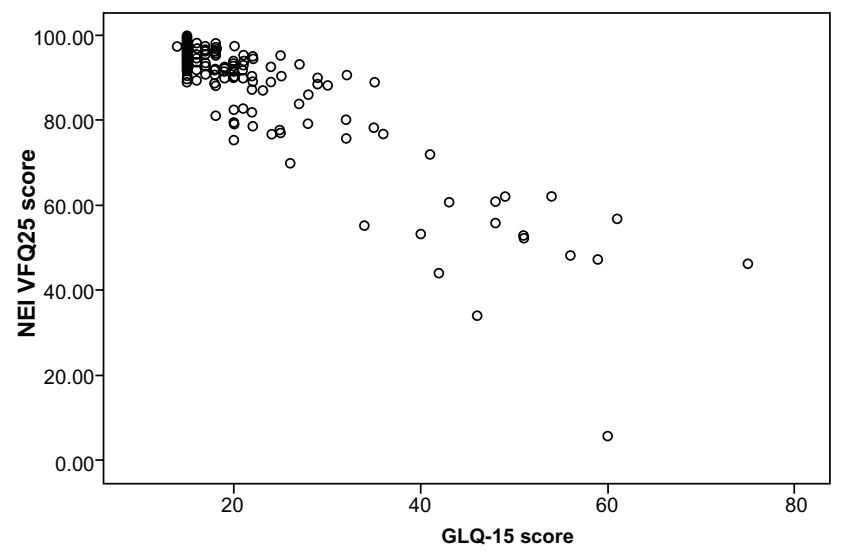

Figure 5 Scatterplot of NEIVFQ25 and GQL-15 scores.

Abbreviations: NEIVFQ25, 25-item National Eye Institute Visual Function Questionnaire; GQL-I5, I5-item Glaucoma Quality of Life questionnaire. that obtained for the NEIVFQ25 (0.93) but is very close to that obtained by Nelson et al ${ }^{16}$ for the GQL-15 (0.95).

In this study, QOL worsened with increasing severity of glaucoma. This has been reported by other researchers. ${ }^{16,21-22,29-32}$ A combination of a generic healthrelated QOL tool and a vision-specific questionnaire was used in some of the studies, ${ }^{21,30}$ unlike in the present study, in which two vision-specific QOL questionnaires were used.

The NEIVFQ25 and the GQL-15 showed good group validity in that they were able to differentiate between cases and controls. They were also able to discriminate between some patients with different degrees of glaucoma severity. The NEIVFQ25 and GQL-15 QOL scores were significantly different for patients with mild and severe glaucoma as well as for patients with moderate and severe glaucoma. However, the QOL scores obtained for patients with mild glaucoma and moderate glaucoma did not differ significantly either for the NEIVFQ25 $(P=0.92)$ or the GQL-15 $(P=0.25)$.

In a study by Goldberg et al, ${ }^{29}$ mean GQL-15 QOL scores of mild, moderate, and severe glaucoma patients were significantly different. Nelson et al, ${ }^{16}$ on the other hand, found a statistically significant difference in the GQL-15 QOL scores of mild and severe glaucoma patients but did not find a statistically significant difference in scores of patients with mild and moderate glaucoma, as in the present study, or in those with moderate and severe glaucoma. The fact that there is no universally acceptable gold standard for categorizing disease severity in glaucoma could account for this.

The line plot in Figure 1 shows decreasing NEIVFQ25 scores with increasing severity of glaucoma, signifying worsening 
of QOL. This decrease is evident even between controls $(n=132)$ and cases of mild glaucoma $(n=44)$. The plot in Figure 2 shows increasing GQL-15 scores (worse QOL) with increasing severity of disease. There is also a clear decrease in QOL when mild cases are compared to controls. The line plots clearly show that the slope of the plot for the QOL from mild to moderate is much less than the slope of the plot from control to mild or moderate to severe for both questionnaires.

This suggests that the belief that patients with mild glaucoma do not have a reduction in QOL until a visual threshold is reached is probably erroneous and corresponds with recent studies that suggest that QOL is reduced in glaucoma patients who are not even aware that they have the disease and in those who are in early stages of the disease. ${ }^{16,34}$ The Los Angeles Latino Eye Study ${ }^{33}$ findings suggest that there is no normal glaucoma patient. They found that QOL measured by the 25-item Visual Function Questionnaire (VFQ25) and the physical component of the Medical Outcomes Study Short-Form 12 (SF-12) was lower in participants with glaucoma compared to those without. This persisted in $75 \%(n=160)$ of cases who did not know they had glaucoma when answering the questions, and therefore, the reduced QOL was clearly a disease effect.

This finding that QOL is reduced significantly in patients with mild glaucoma may also explain why the questionnaires used in this study (NEIVFQ25 and GQL-15) could not differentiate between moderate and mild glaucoma patients. This finding may not be a flaw or weakness in these questionnaires but perhaps suggests that the absence of a significant difference in the QOL of these two groups of patients is because the decrease in QOL had occurred earlier. This suggests that assessment of QOL should be done at the onset of the disease whether or not there is documentation of visual loss (preperimetric glaucoma).

\section{NEIVFQ25, GQL- 15 scores and correlation with visual function}

Both questionnaires correlated only weakly with the PSD visual field index and had a moderate correlation with the MD OU and MD worse eye. The rho value for the correlation between the NEIVFQ25 and the MD worse eye was $0.38(P<0.001)$, and it was $-0.38(P<0.001)$ for the GQL-15. For MD OU, the rho for the NEIVFQ25 was 0.41 $(P<0.001)$ while for the GQL-15, it was $0.43(P<0.001)$. This shows that both questionnaires appeared to correlate nearly equally with the visual field indices in the present study. This differs from the finding of Pourjavan et $\mathrm{al}^{35}$ in which the NEIVFQ25 had a stronger correlation with visual field indices (rho: $0.61,0.55$ ) than the GQL-15, which had a moderate correlation only (rho: $-0.47,0.38$ ).

\section{Correlation between NEIVFQ25 and GQL-I5 scores}

There was a strong correlation between the overall scores of both questionnaires (rho: $-0.753, P<0.001$ ) and both questionnaires showed statistically significant correlations between their subscales/domains. It is important to note that the outdoor mobility domain of the GQL-15 had the highest correlation with the peripheral vision subscale of the NEIVFQ25 (rho: $-0.620, P<0.001$ ) and that the GQL-15 central and near vision domain correlated best with the NEIVFQ25 near activities subscale (rho: $-0.731, P<0.001$ ). Similarly, the GQL-15 peripheral vision domain had a strong correlation (rho: -0.639 , $P<0.001)$ with the NEIVFQ25 peripheral vision subscale. This suggests a high degree of correlation between similar domains or subscales of both instruments.

The NEIVFQ25 driving subscale had the highest correlation with the GQL-15 glare and dark adaptation domain. This is important, as the participants had the greatest difficulty carrying out tasks that were affected by glare and poor dark adaptation such as general vision tasks and driving. One can therefore infer that glare and poor adaptation to darkness may account for some of the difficulties glaucoma patients experience with driving. Therefore, its assessment should be included in the clinical management of glaucoma patients and steps taken to reduce optic glare in the patient's work and home environments and by environmental modification.

Glare and dark adaptation subscale scores of the GQL-15 increased with increasing severity of disease (mild: $11.46 \pm 15.12$; moderate: $11.98 \pm 18.62$; severe: $36.18 \pm 28.74)$ implying increasing difficulty with visual tasks affected by glare and dark adaptation with progression of glaucoma. The glare and dark adaptation score had a moderate correlation with the MD (rho: 0.374).

The fact that there were an equal number of controls and cases as well as an equal number of cases with mild, moderate, or severe glaucoma strengthened the design of this study. Though only eight participants $(3 \%)$ responded to the translated version of the GQL-15 and the NEIVFQ25 questionnaires, which have not been validated, the entire data was re-analyzed excluding these eight participants. A comparison showed no significant difference in the results after excluding these participants so the study results are presented without excluding them. The authors plan to validate these versions in a subsequent multicenter study. 


\section{Conclusion}

The NEIVFQ25 and the GQL-15 both appeared reliable in the assessment of QOL in this study. They showed similarities in their correlation with visual function, visual field indices, and their ability to differentiate between patients with varying severity of glaucoma. The NEIVFQ25, however, is a broader instrument better evaluating psychosocial wellbeing than the GQL-15, while the GQL-15 is a shorter, simpler instrument, which could be easily administered in clinical practice. More studies may further assess the QOL of Nigerian glaucoma patients and the reliability and validity of available QOL instruments in the Nigerian setting. However, it should be noted that, though useful and invaluable, QOL assessment is complementary to clinical evaluation of glaucoma patients and cannot replace it.

\section{Disclosure}

The authors report no conflict of interest in this work.

\section{References}

1. Anonymous. Study protocol for the World Health Organization project to develop a Quality of Life assessment instrument (WHOQOL). Qual Life Res. 1993;2:153-159.

2. Nah YS, Seong GJ, Kin CY. Visual function and QOL in Korean patients with glaucoma. Korean J Ophthalmol. 2002;16:70-74.

3. Massof RW, Rubin GS. Visual function assessment questionnaires. Surv Ophthalmol. 2001;45(6):531-548.

4. Spaeth G, Walt J, Keener J. Evaluation of quality of life for patients with glaucoma. Am J Ophthalmol. 2006;141(Suppl 1):S1-S14.

5. Sawada H, Fukuchi T, Abe H. Evaluation of the relationship between quality of vision and visual function in Japanese glaucoma patients. Clin Ophthalmol. 2011;5:259-267.

6. Cassel EJ. The nature of suffering and the goals of medicine. $N$ Engl J Med. 1982;306:639-645.

7. Patrick DL, Deyo R. Generic and disease-specific measures in assessing health status and quality of life. Med Care. 1989;27 Suppl: S217-S232.

8. Stewart AL, Sherbourne C, Hays RD, et al. Measuring Functioning and Well-Being: The Medical Outcomes Study Approach. Durham, NC: Duke University Press. 1992:345-371.

9. Bergner M, Bobbitt RA, Carter WB, Gilson BS. The Sickness Impact Profile: development and final revision of a health status measure. Med Care. 1981;19(8):787-805.

10. Mangione CM, Lee PP, Pitts J, Gutierrez P, Berry S, Hays RR. The NEIVFQ Field Test Investigators. Psychometric properties of the National Eye Institute Visual Function Questionnaire (NEIVFQ). Arch Ophthal. 1998;116(11):1446-1504.

11. Mangione CM, Lee PP, Pitts J, Gutierrez P, Berry S, Hays RR. The NEIVFQ Field Test Investigators. Development of the 25-item National Eye Institute Visual Function Questionnaire (NEIVFQ). Arch Ophthal. 2001;119(7):1050-1058.

12. Steinberg EP, Tielsch JM, Schein OD, et al. The VF-14: an index of functional impairment in patients with cataract. Arch Ophthal. 1994;112(5):630-638.

13. Altarangel U, Spaeth GL, Steinmann WC. Assessment of function related to vision (AFREV). Ophthalmic Epidemiol. 2006;13(1):67-80.

14. Mangione CM, Phillips RS, Seddon JM, et al. Development of the 'Activities of Daily Vision Scale': a measure of visual functional status. Med Care. 1992;30:11-126.
15. Sloane ME, Ball K, Owsley C, Bruni JR, Roenker DL. The Visual Activities Questionnaire: Developing an instrument for assessing problems in everyday visual tasks. Tech Dig Noninvasive Assess Vis Sys. 1992;1:26-29.

16. Nelson P, Aspinall P, Papasoulitis 0, Worton B, O'Brien C. Quality of life in glaucoma and its relationship with visual function. J Glaucoma. 2003;12(2):139-150.

17. Janz NK, Wren PA, Lichter PR, Musch DC, Gillespie BW, Guire KE. Quality of life in newly diagnosed glaucoma patients: the Collaborative Initial Glaucoma Treatment Study. Ophthalmology. 2001;108(5):887-897.

18. Lee BL, Gutierrez P, Gordon M, et al. The Glaucoma Symptom Scale. A brief index of glaucoma-specific symptoms. Arch Ophthal. Jul 1998;116(7):861-866.

19. Barber BL, Strahlman ER, Laibovitz R, Guess HA, Reines SA. Validation of a questionnaire for comparing the tolerability of ophthalmic medication. Ophthalmology. 1997;104(2):334-341.

20. Mitchell J, Bradley C. Design of an individualized measure of the impact of macular disease on quality of life (the MacDQoL). Qual Life Res. 2004;13:1163-1175.

21. Gutierrez P, Wilson MR, Johnson C, et al. Influence of glaucomatous visual field loss on health-related quality of life. Arch Ophthalmol. 1997;115:777-784.

22. Lester M, Zingirian M. Quality of life in patients with early, moderate and advanced glaucoma. Eye (Lond). 2002;16(1):44-49.

23. Severn P, Fraser S, Finch T, May C. Which quality of life score is best for glaucoma patients and why? BMC Ophthalmol. 2008 [online] [cited April 11, 2008]. Available from: http://www.biomedcentral. com/1471-2415/8/2. Accessed April 13, 2009.

24. Majekodunmi S. Glaucoma in Lagos. Ghana Med J. 1978;17:23-26.

25. Omoti AE. Glaucoma in Benin City, Nigeria. Niger Postgrad Med J. 2005;12:189-192.

26. Enock ME, Omoti AE, Momoh RO. Glaucoma in a Suburban Tertiary Care Hospital in Nigeria. J Ophthalmic Vis Res. 2010;5(2):87-91.

27. Hodapp E, Parrish RK II, Anderson DR. Clinical Decisions in Glaucoma. St Louis: Mosby; 1993:53-61.

28. Chylack LT Jr, Wolfe JK, Singer DM, et al. The Lens Opacities Classification System III. The Longitudinal Study of Cataract Study Group. Arch Ophthalmol. 1993;111:831-836.

29. Goldberg I, Clement CI, Chiang TH, et al. Assessing quality of life in patients with glaucoma using the GQL-15 questionnaire. J Glaucoma. 2009; 18(1):6-12.

30. Parrish RK II, Gedde SJ, Scott IU, et al. Visual function and quality of life in patients with glaucoma. Arch Ophthalmol. 1997;115(11): $1447-1455$.

31. Sherwood MB, Garcia-Siekavizza A, Meltzer MI, Herbert A, Burns AF, McGorray S. Glaucoma's impact on quality of life and its relation to clinical indicators. A pilot study. Ophthalmology. 1998;105:561-566.

32. Skalicky S, Goldberg I. Depression and quality of life in patients with glaucoma: A cross-sectional analysis using the Geriatric Depression Scale-15, assessment of function related to vision and the Glaucoma Quality of Life-15. J Glaucoma. 2008;17(7):546-551.

33. McKean-Cowdin R, Wang Y, Wu J, Asen SP, Varma R; and the Los Angeles Latino Eye Study Group. Impact of visual field loss on health-related QOL in glaucoma. The Los Angeles Latino Eye Study. Ophthalmol. 2008;115(6):941-948.

34. Odberg T, Jakobsen JE, Hultgren SJ, Halseide R. The impact of glaucoma on the quality of life of patients in Norway. I. Results from a self-administered questionnaire. Acta Ophthalmol Scand. 2001;79(2):116-120.

35. Pourjavan S, Spratt A, Kotecha A. Patient reported outcomes in glaucoma: associations between the NEI VFQ-25 and the GQL-15 and clinical measures of visual function. Acta Ophthalmol. 2010;88:0. doi: 10.1111/j.1755-3768.4356.x Accessed October 10, 2009. 
Clinical Ophthalmology

\section{Publish your work in this journal}

Clinical Ophthalmology is an international, peer-reviewed journal covering all subspecialties within ophthalmology. Key topics include: Optometry; Visual science; Pharmacology and drug therapy in eye diseases; Basic Sciences; Primary and Secondary eye care; Patien Safety and Quality of Care Improvements. This journal is indexed on

PubMed Central and CAS, and is the official journal of The Society of Clinical Ophthalmology (SCO). The manuscript management system is completely online and includes a very quick and fair peer-review system, which is all easy to use. Visit http://www.dovepress.com/ testimonials.php to read real quotes from published authors. 